The Astrophysical Journal, 523:L121-L124, 1999 October 1

(C) 1999. The American Astronomical Society. All rights reserved. Printed in U.S.A.

\title{
OPTICAL AND RADIO OBSERVATIONS OF THE AFTERGLOW FROM GRB 990510: EVIDENCE FOR A JET
}

\author{
F. A. Harrison, ${ }^{1}$ J. S. Bloom, ${ }^{1}$ D. A. Frail, ${ }^{2}$ R. Sari, ${ }^{3}$ S. R. Kulkarni, ${ }^{1}$ S. G. Djorgovski, ${ }^{1}$ T. Axelrod, ${ }^{4}$ J. Mould, ${ }^{4}$ \\ B. P. Schmidt, ${ }^{4}$ M. H. Wieringa, ${ }^{5}$ R. M. Wark, ${ }^{5}$ R. Subrahmanyan, ${ }^{5}$ D. McConnell, ${ }^{5}$ P. J. McCarthy, ${ }^{6}$ \\ B. E. Schaefer, ${ }^{7}$ R. G. McMahon, ${ }^{8}$ R. O. Markze, ${ }^{6}$ E. Firth, ${ }^{8}$ P. Soffitta, ${ }^{9}$ and L. Amati ${ }^{10}$ \\ Received 1999 July 12; accepted 1999 August 2; published 1999 September 7
}

\begin{abstract}
We present multicolor optical and two-frequency radio observations of the bright BeppoSAX event GRB 990510. Neither the well-sampled optical decay nor the radio observations are consistent with simple spherical afterglow models. The achromatic steepening in the optical band and the early decay of the radio afterglow, which both occur at $t \sim 1$ day, are evidence for hydrodynamical evolution of the source and can be most easily interpreted by models in which the gamma-ray burst ejecta are collimated in a jet. Employing a simple jet model to explain the observations, we derive a jet opening angle of $\theta_{0}=0.08\left(n / 1 \mathrm{~cm}^{-3}\right)^{1 / 8}$, reducing the isotropic gamma-ray energy release of $2.9 \times 10^{53} \mathrm{ergs}$ by a factor of $\sim 300$.
\end{abstract}

Subject headings: cosmology: miscellaneous — gamma rays: bursts — radio continuum: general — shock waves

\section{INTRODUCTION}

Gamma-ray burst (GRB) afterglow observations from X-ray through radio can be interpreted in the context of fireball models, where a shock produced by the interaction of relativistic ejecta with the circumburst environment expands into the surrounding medium, producing broadband synchrotron emission (e.g., Mészáros \& Rees 1997; Sari, Piran, \& Narayan 1998; Waxman 1997). The optical light curve of GRB 970508, for example, exhibits a monotonic decay: $F_{\nu} \propto t^{-\alpha}$ with $\alpha=1.3$ for 200 days (Fruchter et al. 1999a), well-described by the expansion of a spherical blast wave (Wijers, Rees, \& Mészáros 1997). Recently, the rapid decay of some events has been interpreted as evidence for jetlike or collimated ejecta (Sari, Piran, \& Halpern 1999), but this explanation is not unique (Chevalier \& Li 1999). For GRB 990123, the steepening of the optical light curve (Kulkarni et al. 1999a; Fruchter et al. 1999b) combined with the early radio decay (Kulkarni et al. 1999b) together provide the best evidence to date for deviations from spherical symmetry. Due to sparse sampling, however, simultaneous steepening in all optical bands - the distinctive feature of hydrodynamic evolution of a jet-was not clearly observed.

The bright BeppoSAX event GRB 990510 is distinguished by excellent sampling of the optical decay in multiple bands and by the early-time detection and continued monitoring of the radio afterglow. In this Letter we present the optical and radio light curves and argue that in concert they provide clear

${ }^{1}$ Palomar Observatory 105-24, California Institute of Technology, Pasadena, CA 91125 .

${ }^{2}$ National Radio Astronomy Observatory, Socorro, NM 87801.

${ }^{3}$ California Institute of Technology, Theoretical Astrophysics 103-33, Pasadena, CA 91125.

${ }^{4}$ Research School of Astronomy, Australian National University, Private Bag, Weston Creek P.O., ACT 2611, Australia.

${ }^{5}$ Australian Telescope National Facility, CSIRO, Epping, NSW 2121, Australia.

${ }^{6}$ Observatories of the Carnegie Institute of Washington, 813 Santa Barbara Street, Pasadena, CA 91101-1292.

${ }^{7}$ Department of Physics, Yale University, New Haven, CT 06520.

${ }^{8}$ Institute of Astronomy, Madingley Road, Cambridge CB3 OHA, England, UK.

${ }^{9}$ Istituto Astrofisica Spaziale, CNR, Area di Ricerca Tor Vergata, Via Fosso del Cavaliere 100, 00133 Roma, Italy.

${ }^{10}$ Istituto Tecnologie e Studio Radiazioni Extraterrestri, CNR, Via Gobetti 101, 40129 Bologna, Italy. evidence for a relatively simple jetlike evolution of the ejecta. The level of collimation implied for this event reduces, by a factor greater than 100, the energy required to produce the gamma-ray flash.

\section{OBSERVATIONS}

GRB 990510, imaged by the BeppoSAX Wide Field Camera (WFC) on May 10.37 (UT) (Dadina et al. 1999), was a long $(\sim 75 \mathrm{~s})$ relatively bright event with a fluence $(E>20 \mathrm{keV})$ of $2.6 \times 10^{-5}$ ergs $\mathrm{cm}^{-2}$, ranking it fourth among the BeppoSAX WFC localized sample and in the top $10 \%$ of BATSE bursts (Kippen et al. 1999; Amati et al. 1999). ${ }^{11}$ After announcement of the WFC position by the BeppoSAX team, numerous groups began the search for an optical transient, eventually discovered by Vreeswijk et al. (1999a). The optical transient is coincident with a fading X-ray source seen in the BeppoSAX Narrow Field Instruments (Kuulkers et al. 1999). Spectra taken with the VLT (Vreeswijk et al. 1999b) identify numerous absorption lines, determining a minimum redshift of $1.619 \pm 0.002$. Adopting this as the source redshift implies an isotropic gamma-ray energy release of $2.9 \times 10^{53}$ ergs (we employ a standard Friedmann cosmology with $H_{0}=65 \mathrm{~km} \mathrm{~s}^{-1} \mathrm{Mpc}^{-1}, \Omega_{0}=0.2$, and $\Lambda=0$ throughout).

We commenced optical observations of the $3^{\prime}$ radius BeppoSAX WFC error circle using the Mount Stromlo Observatory (MSO) 50 inch $(1.3 \mathrm{~m})$ telescope $3.5 \mathrm{hr}$ after the GRB. We continued monitoring with the MSO 50 inch, the Yale $1 \mathrm{~m}$ telescope on Cerro Tololo in Chile, and the 40 inch $(1 \mathrm{~m})$ telescope at the Las Campanas Observatory (LCO) in Chile. Radio observations began at the Australia Telescope Compact Array (ATCA), in Narrabri, Australia, about $17 \mathrm{hr}$ following the GRB event. Tables 1, 2, 3, and 4 present the BVRI optical data taken by our collaboration (quoted errors are $1 \sigma$ statistical uncertainties). The $V R$ and $I$ light curves, along with points from numerous other groups reported in the literature (Galama et al. 1999; Kaluzny et al. 1999; Stanek et al. 1999a; Pietrzynski \& Udalski 1999a, 1999b; Covino et al. 1999; Lazzati, Covino, \& Ghisellini 1999; Pietrzynski \& Udalski 1999c; Marconi et al. 1999), are plotted in Figure 1. We have calibrated the re-

\footnotetext{
${ }^{11}$ GCN circulars are available at http://lheawww.gsfc.nasa.gov/docs/gamcosray/legr/bacodine/gcn_main.html.
} 
TABLE 1

$B$-BAND Photometry OF GRB 990510

\begin{tabular}{ccc}
\hline \hline $\begin{array}{c}\text { Date } \\
(1999 \text { May UT })\end{array}$ & Magnitude & Telescope \\
\hline $10.971 \ldots \ldots \ldots$ & $19.86 \pm 0.05$ & Yale $1 \mathrm{~m}$ \\
$11.058 \ldots \ldots \ldots$ & $17.88 \pm 0.05$ & Yale $1 \mathrm{~m}$ \\
$11.131 \ldots \ldots \ldots$ & $17.95 \pm 0.05$ & Yale $1 \mathrm{~m}$ \\
$11.154 \ldots \ldots \ldots$ & $18.84 \pm 0.06$ & Yale $1 \mathrm{~m}$ \\
$11.180 \ldots \ldots \ldots$ & $18.90 \pm 0.06$ & Yale $1 \mathrm{~m}$ \\
$11.207 \ldots \ldots \ldots$ & $18.98 \pm 0.06$ & Yale $1 \mathrm{~m}$ \\
$11.266 \ldots \ldots \ldots$. & $19.23 \pm 0.06$ & Yale $1 \mathrm{~m}$ \\
$11.292 \ldots \ldots \ldots$ & $19.39 \pm 0.06$ & Yale $1 \mathrm{~m}$ \\
$11.320 \ldots \ldots \ldots$ & $20.11 \pm 0.06$ & Yale $1 \mathrm{~m}$ \\
$12.125 \ldots \ldots \ldots$ & $20.01 \pm 0.08$ & Yale $1 \mathrm{~m}$ \\
$12.171 \ldots \ldots \ldots$ & $20.06 \pm 0.09$ & Yale $1 \mathrm{~m}$ \\
$12.221 \ldots \ldots \ldots$ & $20.89 \pm 0.09$ & Yale $1 \mathrm{~m}$ \\
$12.300 \ldots \ldots \ldots$ & $21.22 \pm 0.12$ & Yale $1 \mathrm{~m}$ \\
$12.996 \ldots \ldots \ldots$ & $21.22 \pm 0.17$ & Yale $1 \mathrm{~m}$ \\
\hline
\end{tabular}

ported magnitudes to the Landolt bandpass system (approximately Johnson-Cousins). For calibration, we observed a number of Landolt stars on May 11 under photometric conditions with the MSO 50 inch telescope. The uncertainty in the zero point of the calibration introduces a magnitude error of \pm 0.03 in all bands.

From Figure 1, it is evident that the light curve steepens contemporaneously in all bands between day 1 and 2. To characterize the shape, we fit the data with the following analytic four-parameter function:

$$
\begin{gathered}
F_{\nu}(t)=f_{*}\left(t / t_{*}\right)^{\alpha_{1}}[1-\exp (-J)] / J ; \\
J\left(t, t_{*}, \alpha_{1}, \alpha_{2}\right)=\left(t / t_{*}\right)^{\left(\alpha_{1}-\alpha_{2}\right)} .
\end{gathered}
$$

The functional form has no physical significance, but provides a good description of the data and has the property that the asymptotic power-law indices are $\alpha_{1}$ and $\alpha_{2}$ at early and late times, respectively. Fitting the $V, R$, and $I$ data (excluding $B$ due to larger statistical uncertainties) simultaneously yields $t_{*}=1.20 \pm 0.08$ days, $\alpha_{1}=-0.82 \pm 0.02$, and $\alpha_{2}=-2.18$ \pm 0.05 , where the errors are formal $1 \sigma$ errors and do not reflect the covariance between parameters. The $\chi^{2}$ for the fit is acceptable: 65 for 82 degrees of freedom. We have removed five out of the 92 total data points with uncertain calibrations. Due to calibration uncertainty, we cannot determine if the light curve exhibits variability on timescales shorter than the trend described by the functional fit. The difference in fit parameters from those found by Stanek et al. (1999b) is due to the slightly

TABLE 2

$V$-BAnd Рhotometry of GRB 990510

\begin{tabular}{lcl}
\hline \hline $\begin{array}{c}\text { Date } \\
(1999 \text { May UT })\end{array}$ & Magnitude & Telescope \\
\hline $10.514 \ldots \ldots \ldots$ & $17.84 \pm 0.02$ & MSO 50 inch \\
$10.522 \ldots \ldots \ldots$ & $17.88 \pm 0.02$ & MSO 50 inch \\
$10.529 \ldots \ldots \ldots$ & $17.95 \pm 0.01$ & MSO 50 inch \\
$10.775 \ldots \ldots \ldots$ & $18.84 \pm 0.06$ & MSO 50 inch \\
$10.783 \ldots \ldots \ldots$ & $18.90 \pm 0.08$ & MSO 50 inch \\
$10.791 \ldots \ldots \ldots$ & $18.98 \pm 0.05$ & MSO 50 inch \\
$10.979 \ldots \ldots \ldots$ & $19.23 \pm 0.04$ & Yale 1 m \\
$11.011 \ldots \ldots \ldots$ & $19.39 \pm 0.05$ & LCO 40 inch \\
$11.508 \ldots \ldots \ldots$ & $20.11 \pm 0.09$ & MSO 50 inch \\
$11.512 \ldots \ldots \ldots$. & $20.01 \pm 0.08$ & MSO 50 inch \\
$11.516 \ldots \ldots \ldots$. & $20.06 \pm 0.07$ & MSO 50 inch \\
$12.146 \ldots \ldots \ldots$ & $20.89 \pm 0.07$ & Yale 1 m \\
$12.367 \ldots \ldots \ldots$ & $21.22 \pm 0.14$ & LCO 40 inch \\
\hline
\end{tabular}

TABLE 3

\begin{tabular}{|c|c|c|}
\hline $\begin{array}{c}\text { Date } \\
\text { (1999 May UT) }\end{array}$ & Magnitude & Telescope \\
\hline 10.514 & $17.54 \pm 0.02$ & MSO $50 \mathrm{inch}$ \\
\hline 10.522 & $17.61 \pm 0.02$ & MSO 50 inch \\
\hline 10.529 & $17.60 \pm 0.02$ & MSO 50 inch \\
\hline 10.775 & $18.53 \pm 0.07$ & MSO 50 inch \\
\hline 10.783 & $18.61 \pm 0.07$ & MSO 50 inch \\
\hline 10.791 & $18.55 \pm 0.04$ & MSO 50 inch \\
\hline 10.992 & $18.90 \pm 0.04$ & Yale $1 \mathrm{~m}$ \\
\hline 11.071 & $19.07 \pm 0.04$ & Yale $1 \mathrm{~m}$ \\
\hline 11.094 & $19.20 \pm 0.04$ & Yale $1 \mathrm{~m}$ \\
\hline 11.194 & $19.24 \pm 0.04$ & Yale $1 \mathrm{~m}$ \\
\hline 11.280 & $19.35 \pm 0.05$ & Yale $1 \mathrm{~m}$ \\
\hline $11.333 \ldots$ & $19.40 \pm 0.06$ & Yale $1 \mathrm{~m}$ \\
\hline 11.508 & $19.67 \pm 0.07$ & MSO 50 inch \\
\hline 11.512 . & $19.71 \pm 0.06$ & MSO 50 inch \\
\hline $11.516 \ldots \ldots \ldots$ & $19.76 \pm 0.09$ & MSO 50 inch \\
\hline 12.138 . & $20.49 \pm 0.08$ & Yale $1 \mathrm{~m}$ \\
\hline $12.183 \ldots$ & $20.59 \pm 0.09$ & Yale $1 \mathrm{~m}$ \\
\hline $12.233 \ldots \ldots$ & $20.47 \pm 0.12$ & Yale $1 \mathrm{~m}$ \\
\hline $12.975 \ldots \ldots \ldots$ & $21.04 \pm 0.14$ & Yale $1 \mathrm{~m}$ \\
\hline $13.238 \ldots \ldots \ldots$ & $21.42 \pm 0.14$ & Yale $1 \mathrm{~m}$ \\
\hline $14.308 \quad \ldots \ldots \ldots$ & $22.01 \pm 0.18$ & Yale $1 \mathrm{~m}$ \\
\hline
\end{tabular}

$R$-BANd РнOTOMETRY OF GRB 990510

different function used. Using the same function, we find consistency with their results to better than $2 \sigma$ in all parameters.

To derive the extinction-corrected normalizations, obtained by fitting with the shape described above, we use the astrometric position from Hjorth et al. (1999) [R.A. $=13^{\mathrm{h}} 38^{\mathrm{m}} 07^{\mathrm{s}} .11$, decl. $=-80^{\circ} 29^{\prime} 48^{\prime \prime 2}$ (J2000)] and the dust maps from Schegel, Finkbeiner, \& Davis (1998). The resulting Galactic extinction in the direction of the transient is $E(B-V)=0.20$. In the standard Landolt bandpass system, assuming $R_{V}=A_{V} / E(B-V)=$ 3.1, we obtain $A_{B}=0.87, A_{V}=0.67, A_{R}=0.54$, and $A_{I}=0.40$. After correction, the magnitudes corresponding to the flux $f_{*}$ in equation (1) are $V_{*}=19.03 \pm 0.01, I_{*}=18.42 \pm 0.01$, and $R_{*}=18.81 \pm 0.01$. The errors are the formal $1 \sigma$ errors from the fit, with an additional \pm 0.03 mag due to the uncertain zero-point calibration.

Observations of the field around GRB 990510 with ATCA began on 1999 May 10 at 22:36 UT. All observations (Table 5) use a bandwidth of $128 \mathrm{MHz}$ and two orthogonal linear polarizations for each wavelength pair. A radio afterglow is clearly detected, starting $\sim 3$ days after the event (Fig. 2). The error bars provided in the table are statistical (radiometric) errors only. At early times, variation due to interstellar scintillation will dominate the error in flux determination from the source (see the legend to Fig. 2).

\section{EVIDENCE FOR A JET}

The majority of other well-studied GRBs, in particular GRB 970228 and GRB 970508, have afterglow light curves that decay monotonically for the first month or more, and these have been interpreted in the context of spherical fireball models (e.g., Tavani 1997; Wijers et al. 1997; Reichart 1997; Granot,

TABLE 4

I-BAND PHOTOMETRY OF GRB 990510

\begin{tabular}{lcl}
\hline \hline $\begin{array}{c}\text { Date } \\
(1999 \text { May UT) }\end{array}$ & Magnitude & Telescope \\
\hline $10.999 \ldots \ldots \ldots$ & $18.40 \pm 0.04$ & Yale $1 \mathrm{~m}$ \\
$12.154 \ldots \ldots \ldots$ & $20.04 \pm 0.09$ & Yale $1 \mathrm{~m}$ \\
$11.034 \ldots \ldots \ldots$ & $18.61 \pm 0.05$ & LCO 40 inch \\
$12.042 \ldots \ldots \ldots$ & $19.83 \pm 0.10$ & LCO 40 inch \\
\hline
\end{tabular}




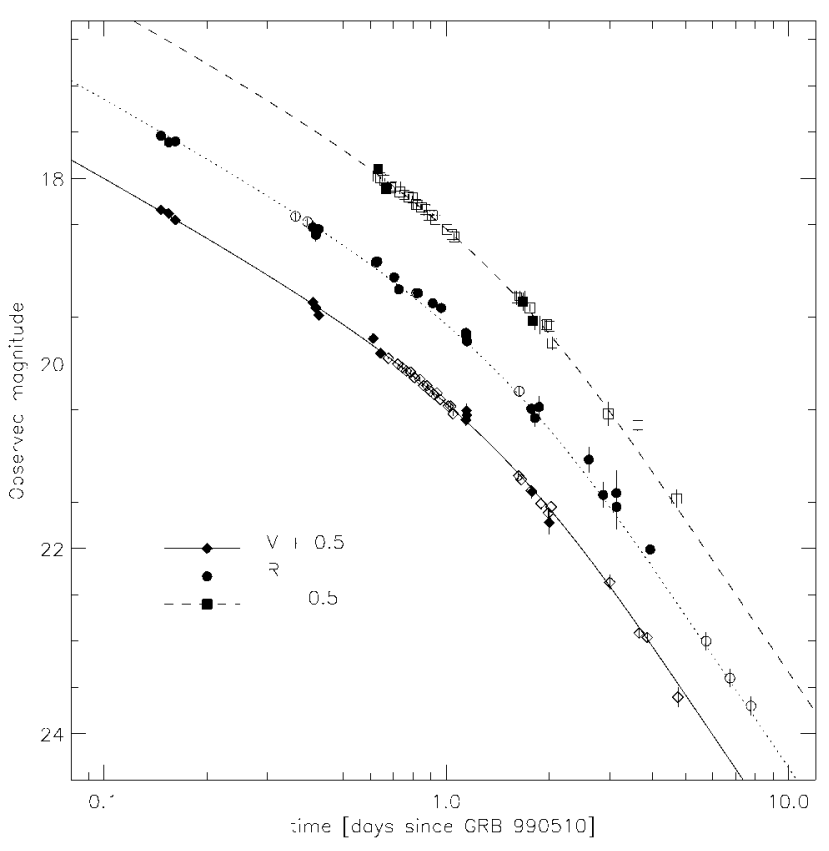

FIG. 1.-Optical light curves of the transient afterglow of GRB 990510. In addition to photometry from our group (filled symbols; see Table 1), we have augmented the light curves with data from the literature (open symbols). The photometric zero points in Landolt $V$-band from our group are consistent with that of the OGLE group (Pietrzynski \& Udalski 1999b), and the $I$-band zero point is from the OGLE group. Some $R$-band measurements were based on an incorrect calibration of a secondary star in the field (Galama et al. 1999), and we have recalibrated these measurements.

Piran, \& Sari 1999). In the optical, spherical models with typical parameters predict flux rising quickly (within hours) to a maximum value $f_{m}$ (at time $t_{m}$ ), after which it decays as a power law, $t^{-\alpha}$ with $\alpha \sim 1$. At later times, the decay becomes somewhat faster (a change in $\alpha$ of 0.25 ), as the cooling break sweeps across the band (Sari, Piran, \& Narayan 1998). In the radio band, above the self-absorption frequency, the behavior is similar, but with typical values of $t_{m} \sim 1$ week.

The observed optical and radio decay of GRB 990510 is quite distinct, showing frequency-independent steepening in the optical and early decline in the radio on a timescale of 1 day-behavior clearly inconsistent with spherical models. An achromatic break or steepening in light curves is expected if the emitting surface has a nonspherical geometry. At any given time, due to relativistic beaming, only a small portion of the emitting surface with opening angle $1 / \gamma$ is visible. At early times (when $\theta_{0} \gtrsim 1 / \gamma$ ), the observed light curve from a collimated source is identical to that of a sphere. As the fireball evolves and $\gamma$ decreases, the beaming angle will eventually exceed the opening angle of the jet, and we expect to see a deficit in the emission-i.e., a break in the light curve. At a comparable or later time (Rhoads 1999; Sari, Piran, \& Halpern 1999; Panaitescu, Mészáros, \& Rees 1998) the jet will begin to spread laterally, causing a further steepening.

To model the light curve, we adopt the afterglow analysis for a jet source given in Sari et al. (1999). At early times $\left(\gamma>\theta_{0}^{-1}\right)$, the light curve is given by the spherical solution; $F\left(\nu_{0}\right) \propto t^{\alpha}$ with $\alpha=-3(p-1) / 4$ if the electrons are not cooling, and $\alpha=-3 p / 4+1 / 2$ if they are. From the GRB 990510 early-time optical slope, $\alpha_{1}=-0.82$, and we derive $p=2.1$ assuming the electrons producing the optical emission are in the slow cooling regime and $p=1.76$ otherwise. The latter
TABLE 5

atCa Radio Flux Measurements

\begin{tabular}{ccccc}
\hline \hline $\begin{array}{c}\text { Date } \\
(1999 \text { May UT) }\end{array}$ & $\begin{array}{c}\text { Frequency } \\
(\mathrm{GHz})\end{array}$ & $\begin{array}{c}\text { Flux } \\
\text { Density } \\
(\mu \mathrm{Jy})\end{array}$ & $\begin{array}{c}\text { Integration } \\
(\mathrm{hr})\end{array}$ & $\begin{array}{c}\text { Angular } \\
\text { Resolution } \\
(\operatorname{arcsec})\end{array}$ \\
\hline $11.09 \ldots \ldots \ldots \ldots$ & 4.8 & $110 \pm 69$ & 7.5 & $4.2 \times 1.8$ \\
$11.09 \ldots \ldots \ldots \ldots$ & 8.6 & $104 \pm 74$ & 7.5 & $1.9 \times 1.3$ \\
$13.68 \ldots \ldots \ldots \ldots$ & 8.7 & $227 \pm 30$ & 9.0 & $1.9 \times 1.3$ \\
$15.61 \ldots \ldots \ldots \ldots$ & 8.7 & $202 \pm 31$ & 8.0 & $1.8 \times 1.4$ \\
$17.58 \ldots \ldots \ldots \ldots$ & 8.7 & $138 \pm 32$ & 6.6 & $2.1 \times 1.2$ \\
$19.59 \ldots \ldots \ldots \ldots$ & 4.8 & $177 \pm 36$ & 11.4 & $3.1 \times 2.6$ \\
$19.59 \ldots \ldots \ldots \ldots$ & 8.6 & $127 \pm 31$ & 11.4 & $1.7 \times 1.5$ \\
$25.32 \ldots \ldots \ldots \ldots$ & 8.7 & $82 \pm 32$ & 10.6 & $2.2 \times 1.2$ \\
$46.81 \ldots \ldots \ldots \ldots$ & 8.7 & $-1 \pm 28$ & 11.7 & $4.0 \times 3.6$ \\
\hline
\end{tabular}

Note. - The date indicates the observation center.

value would result in the electron energy being unbounded, and we conclude that $p=2.1$. At late times $\left(\gamma<\theta_{0}^{-1}\right)$, when the evolution is dominated by the spreading of the jet, the model predicts $\alpha=-p$, independent of the cooling regime. Indeed, our measured value of $\alpha_{2}=-2.18 \pm 0.05$ is consistent with this expectation.

The optical data allow us to infer $p$ and the epoch of the break (related to the opening angle of the jet). However, in order to fully characterize the afterglow we also need to determine (1) $\nu_{a}$, the self-absorption frequency, (2) $F_{m}$ and $t_{m}$, and (3) the cooling frequency $\nu_{c}$ at a given epoch. The optical observations show that even at early times the optical flux is decaying and is therefore above $\nu_{m}$. The radio, however, is well below $\nu_{m}$, and by combining the ATCA and optical data we can derive $F_{m}, t_{m}$, and $\nu_{m}$. Following Sari et al. (1999), we have fitted a $t^{-1 / 3}$ power law to the four radio points and obtained $F_{8.7 \mathrm{GHz}} \simeq 204 \mu \mathrm{Jy}\left(t / t_{1}\right)^{-1 / 3}$, where $t_{1}=3.3$ days is the time of

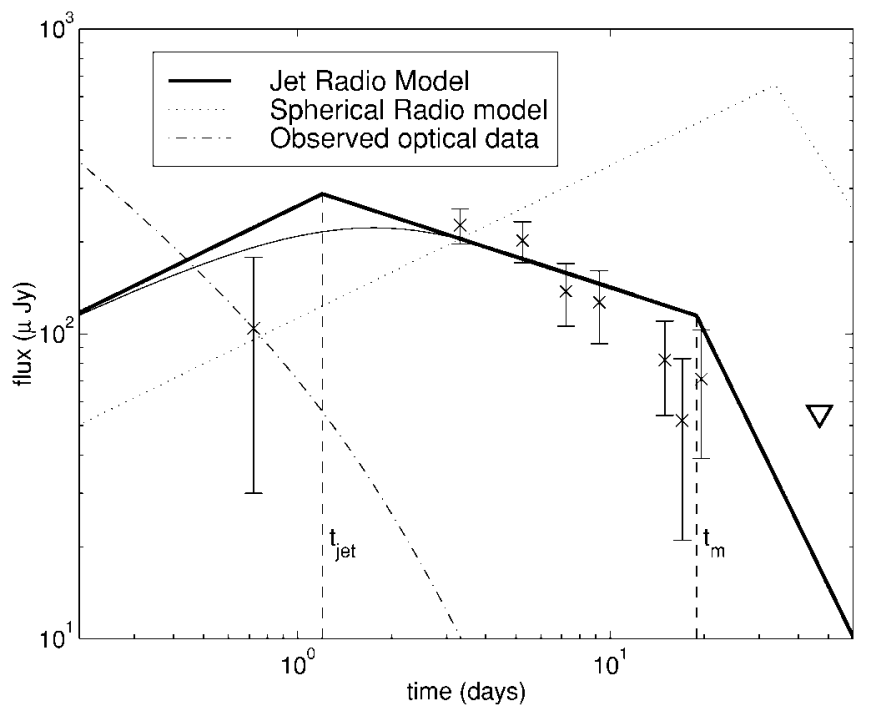

FIG. 2.-Observed and predicted radio light curves at $8.6 \mathrm{GHz}$. Detections are indicated by the crosses, with error bars indicating the rms noise in the image. The true flux uncertainty is dominated by the signal modulation due to refractive interstellar scintillation (e.g., Frail et al. 1997). Using the Galactic scattering model of Taylor \& Cordes (1993) and the formalism from Goodman (1997), we calculate a scintillation timescale of $2 \mathrm{hr}$ in the first few weeks after the burst. Although our typical $8 \mathrm{hr}$ integrations average over the scintillation, we expect modulation of the mean flux density of order $50 \%$. Predictions for the evolution of the radio flux density (solid line) are based on the jet model of Sari et al. (1999) (see text for more details). The dotted line shows the model prediction for a spherical fireball. The dotdashed line illustrates the observed optical behavior. 
the second radio detection. Using this and the optical data at $t_{1}$, we get $\nu_{m}\left(t_{1}\right)=280 \mathrm{GHz}$ and $F_{m}\left(t_{1}\right)=650 \mu \mathrm{Jy}$. After the jet begins to spread, $\nu_{m}$ decays as $t^{-2}$, and we expect $\nu_{m}$ to arrive at radio frequencies at $\sim 19$ days, producing a break in the radio light curve to the $t^{-p}$ slope seen in the optical. In the above, we have assumed that $\nu_{a}$ is below $8.7 \mathrm{GHz}$. A $\chi^{2}$ analysis constrains the $4.8-8.7 \mathrm{GHz}$ spectral slope to be between -1.3 and 0.4 (95\% confidence), consistent with the $\nu^{1 / 3}$ slope expected if $\nu_{a}<8.7 \mathrm{GHz}$ and inconsistent with the $\nu^{2}$ expected if $\nu_{a}>8.7 \mathrm{GHz}$.

Figure 2 shows the radio light curve along with the prediction for both spherical (dotted line) and collimated (solid line) ejecta. The relatively sharp transition in the GRB 990510 decay to the asymptotic value $\alpha_{2}=-p$ expected when both the jet edge becomes visible and when lateral spreading begins suggest both transitions occur at similar times in this event.

Using the gamma-ray energy of $2.9 \times 10^{53} \mathrm{ergs}$, we find a Lorentz factor at the jet break time of $12\left(n / 1 \mathrm{~cm}^{-3}\right)^{-1 / 8}$. This implies an opening angle of $\theta_{0}=0.08\left(n / 1 \mathrm{~cm}^{-3}\right)^{1 / 8}$, and for a two-sided jet the energy is reduced by a factor $2 / \theta_{0}^{2} \cong 300$ to $1 \times 10^{51}\left(\mathrm{n} / 1 \mathrm{~cm}^{-3}\right)^{1 / 4}$ ergs. $^{12}$

\section{CONCLUSION}

With one of the best-sampled optical light curves and simultaneous early-time radio observations, GRB 990510 provides the clearest signature observed to date for collimation of the ejecta in GRB sources. The achromatic steepening in the optical light curve as well as the early decay, after $t \sim 1$ day, of the radio emission is inconsistent with other observed af-

\footnotetext{
${ }^{12}$ The estimates of Rhoads (1999) will give a smaller opening angle and therefore a lower energy; here we have used the estimates in Sari et al. (1999).
}

terglows that have been modeled with spherically symmetric ejecta. The GRB 990510 afterglow emission can be remarkably well fit by a simple model for the jet evolution.

It is interesting to ask if the observations to date are consistent with all GRB engines having an energy release of $\lesssim 10^{52}$ ergs, with the wide observed luminosity distribution being due to variation in the degree of collimation. Of GRBs with measured redshifts for which the gamma-ray energy release can be calculated, only GRB 990123 and GRB 990510 show breaks in the optical light curves on timescales less than 1 week, and interestingly these are among the highest fluence BeppoSAX events to date. GRB 990123 has an implied isotropic energy release of $3.4 \times 10^{54} \mathrm{ergs}$, which reduces by a factor of $\sim 100$ if the light-curve break occurring at $t \sim 2$ days is interpreted as the signature of a jet. As argued here, the energy required for GRB 990510 in the context of the jet model is $\sim 10^{51}$ ergs. In contrast, GRB 970508 and GRB 970228 show no evidence for a jet in the optical (although GRB 970508 may in radio); however, their isotropic energy release is quite modest: only $8 \times 10^{51}$ and $5 \times 10^{51} \mathrm{ergs}$, respectively. The candidates for the largest energy release, highest gamma-ray fluence where no evidence for collimation is seen, are GRB 971214 $(z=3.2)$ with $E_{\gamma}=3 \times 10^{53}$ ergs (Kulkarni et al. 1998) and GRB $980703(z=0.966)$ with $E_{\gamma}=1 \times 10^{53}$ ergs (Djorgovski et al. 1998). Light-curve observations of these events are, however, limited to $t \lesssim 2$ weeks, and so collimation may still reduce the energy of these bursts by factors of $\sim 40$, still consistent with a total energy release $\lesssim 10^{52}$ ergs.

We thank Scott Barthelmy for operating the GCN, the staffs of LCO, MSSSO, and ATCA, and the entire BeppoSAX team. This work was supported by grants from NSF (S. R. K. and S. G. D.), NASA (F. A. H. and S. R. K.), and the Bressler Foundation (S. G. D.).

\section{REFERENCES}

Amati, L., et al. 1999, GCN Circ. 317 (http://gcn.gsfc.nasa.gov/gen/gen3/317 .gcn 3)

Chevalier, R. A., \& Li, Z.-Y. 1999, ApJ, 520, L29

Covino, S., Fugazza, D., Ghisellini, G., \& Lazzati, D. 1999, GCN Circ. 321 (http://gcn.gsfc.nasa.gov/gcn/gcn3/321.gcn3)

Dadina, M., et al. 1999, IAU Circ. 7160

Djorgovski, S. G., Kulkarni, S. R., Bloom, J. S., Goodrich, R., Frail, D. A., Piro, L., \& Palazzi, E. 1998, ApJ, 508, L17

Frail, D. A., Kulkarni, S. R., Nicastro, S. R., Feroci, M., \& Taylor, G. B. 1997, Nature, 389, 261

Fruchter, A. S., et al. 1999a, ApJL, submitted (astro-ph/9903236) 1999b, ApJ, 519, L13

Galama, T. J., et al. 1999, GCN Circ. 313 (http://gcn.gsfc.nasa.gov/gen/gcn3/ 313.gcn3)

Goodman, J. 1997, NewA, 2, 449

Granot, J., Piran, T., \& Sari, R. 1999, ApJ, 513, 679

Hjorth, J., Barud, I., Pizella, A., Pederson, H., Jaunsen, A. O., \& Lindgren, B. 1999, GCN Circ. 320 (http://gcn.gsfc.nasa.gov/gcn/gcn3/320.gcn3)

Kaluzny, J., Garnavich, P. M., Stanek, K. Z., Pych, W., \& Thompson, I. 1999, GCN Circ. 314 (http://gcn.gsfc.nasa.gov/gcn/gcn3/314.gcn3)

Kippen, R. M., et al. 1999, GCN Circ. 322 (http://gcn.gsfc.nasa.gov/gcn/gen3/ 322.gcn3)

Kulkarni, S. R., et al. 1998, Nature, 393, 35 1999a, Nature, 398, 389

1999b, ApJ, submitted (astro-ph/9903441)

Kuulkers, E., et al. 1999, GCN Circ. 326 (http://gcn.gsfc.nasa.gov/gcn/gcn3/ 326.gcn3)
Lazzati, D., Covino, S., \& Ghisellini, G. 1999, GCN Circ. 325 (http://gcn.gsfc .nasa.gov/gcn/gen3/325.gcn3)

Marconi, G., Israel, G. L., Lazzati, D., Covino, S., \& Ghisellini, G. 1999, GCN Circ. 329 (http://gcn.gsfc.nasa.gov/gcn/gcn3/329.gcn3)

Mészáros, P., \& Rees, M. J. 1997, ApJ, 476, 232

Panaitescu, A., Mészáros, P., \& Rees, M. J. 1998, ApJ, 503, 314

Pietrzynski, G., \& Udalski, A. 1999a, GCN Circ. 316 (http://gcn.gsfc.nasa.gov/ $\mathrm{gcn} / \mathrm{gcn} 3 / 316 . \mathrm{gcn} 3)$

. 1999b, GCN Circ. 319 (http://gcn.gsfc.nasa.gov/gcn/gcn3/319.gcn3) 1999c, GCN Circ. 328 (http://gcn.gsfc.nasa.gov/gcn/gcn3/328.gcn3)

Reichart, D. E. 1997, ApJ, 485, L57

Rhoads, J. E. 1999, ApJ, in press (astro-ph/9903399)

Sari, R., Piran, T., \& Halpern, J. P. 1999, ApJ, 519, L17

Sari, R., Piran, T., \& Narayan, R. 1998, ApJ, 497, L17

Schlegel, D. J., Finkbeiner, D. P., \& Davis, M. 1998, ApJ, 500, 525

Stanek, K. Z., Garnavich, P. M., Kaluzny, J., Pych, W., \& Thompson, I. 1999a, GCN Circ. 318 (http://gcn.gsfc.nasa.gov/gcn/gcn3/318.gcn3) 1999b, ApJ, 522, L39

Tavani, M. 1997, ApJ, 483, L87

Taylor, J. H., \& Cordes, J. M. 1993, ApJ, 411, 674

Vreeswijk, P. M., et al. 1999a, GCN Circ. 310 (http://gcn.gsfc.nasa.gov/gcn/ gcn3/310.gcn3) 1999b, GCN Circ. 324 (http://gcn.gsfc.nasa.gov/gcn/gen3/324.gcn3)

Waxman, E. 1997, ApJ, 489, L33

Wijers, R. A. M. J., Rees, M. J., \& Mészáros, P. 1997, MNRAS, 288, L51 